\title{
Regularization and Stabilization of the Rectangle Descriptor Decentralized Control Systems by Dynamic Compensator
}

\author{
Xiumei Tian \\ Department of Electromechanical Engineering, Heze University \\ Heze 274000, China \\ E-mail: showmelwood@163.com
}

Received: December 16, 2010

Accepted: January 17, 2011

doi:10.5539/mas.v5n2p244

\begin{abstract}
The regularization, the impulse-free, and the stabilization of the rectangle descriptor decentralized control system by dynamic compensation are studied in this article. The necessary and sufficient condition of the regularization and the free impulse of the closed-system after compensation, and the necessary and sufficient condition that the rectangle descriptor decentralized control system could make it real stable by the dynamic feedback are provided and the results keep consistent with the square system in form.
\end{abstract}

Keywords: Rectangle descriptor decentralized control systems, Dynamic compensator, Regularization, Impulse-free, Stabilization

\section{Introduction}

In recent years, the research on the descriptor decentralized control system has been developed largely, and especially for the decentralized (pulse) fixed modes and the impulse controllability/ observability, a series of results have occurred (S. H. Wang, 1973, P. 473-478 \& Q. L. Zhang, 1989, P.866-871 \& T.N. Chang, 1986, P.1276-1181 \& T.N. Chang, 2001, P. 1589-1595 \& Xie, 1986, P.185-188 \& Xie, 1995, P.145-153 \& L. Dai, 1989).

Formerly, the coefficient matrix of the system was square, i.e. the line number equaled the column number, but no matter in theory or in practice, the coefficient matrix may be rectangular, for example, the rectangular matrix pencil $(s M-N)$ is used to denote the structure character of the system, and describe some differential equation in the practice, which is very important to study common system. Q. L. Zhang defined the finite fixed modes and the impulse fixed modes of the common descriptor system (including centralized system and decentralized system) (Q. L. Zhang, 1989, P.866-871). Ishihara J Y redefined and studied the pulse controllability and impulse observability of the non-square (rectangular) descriptor system (Ishihara J Y, 2001, P.991-994), and Hou M defined the controllability of another kind of impulse modulus to the non-square descriptor system, and proved this definition was equivalent to eliminating the impulse modulus by feedback (Hou M, 2004, P.1723-1727).

The target of this article is to design $N$ dynamic compensators to make the whole closed-loop systems to be regular, closed, gradually stable, and impulse-free.

\section{Basic knowledge}

For the descriptor decentralized control system with $N$ local control stations described by

$$
\left\{\begin{array}{l}
E \dot{x}(t)=A x(t)+\sum_{i=1}^{N} B_{i} u_{i}(t) \\
y_{i}(t)=C_{i} x(t), i \in \underline{N}=\{1,2, \cdots, N\}
\end{array}\right.
$$

Where $x \in R^{n}$ is the state, $u_{i} \in R^{q_{i}}$ and $y_{i} \in R^{p_{i}}$ are the input and the output, respectively, of the ith local control station, the matrices $E, A \in R^{m \times n}, B_{i} \in R^{m \times q_{i}}, C_{i} \in R^{p_{i} \times n}$ are real and constant, $i \in \underline{N}=\{1,2, \cdots, N\}$. Assume the matrices B,C are full rank, and $\operatorname{rank}(E)=r, 0 \leq r \leq \min \{m, n\}$ obviously. If $m=n$ and $\operatorname{det}(s E-A) \neq 0, s \in c$,we call (1) is regular, otherwise, if $m \neq n$ or $\operatorname{det}(s E-A)=0$, we call (1) is non-regular. If $m=n$, we also call (1) is a square system. If not, we call it a 
rectangular system or a non- square system. We sometimes denote system (1) by $(E, A, B, C)$ for short. For the square system, following lemmas are fundamental.

Lemma 1 (D. Wang, 1989, P.127-131): For the square system (1), the control rules exist, $u_{i}=k_{i} y_{i}+v_{i}, \quad i \in \underline{N}=\{1, \ldots, N\}$, and the sufficient and necessary condition to make the closed-loop systems

$E \dot{x}=\left[A+\sum_{i=1}^{N} B_{i} K_{i} C_{i}\right] x+\sum_{i=1}^{N} B_{i} v_{i}$

, be regular and pulse-free is that for any non-intersect divisions $P=\left\{i_{1}, \cdots, i_{k}\right\}$ and $\bar{P}=\left\{i_{k+1}, \cdots, i_{N}\right\}$ of the set $\underline{N}=\{1,2, \cdots, N\}$,

$\operatorname{rank}\left[\begin{array}{ccc}0 & E & 0 \\ E & A & B_{P} \\ 0 & C_{\bar{P}} & 0\end{array}\right] \geq n+\operatorname{rank}[E]$

Lemma 2 (Hou M, 2004, P.1723-1727): The sufficient and necessary condition to make the square descriptor system $E \dot{x}=A x$ to be regular and impulse-free is

$\operatorname{rank}\left[\begin{array}{cc}0 & E \\ E & A\end{array}\right]=n+\operatorname{rank}[E]$

Lemma 3 (X. K. Xie, 1988, P. 1550-1551): Supposed that $A \in R^{m \times n}, B \in R^{m \times q}$, and $C \in R^{p \times n}$ are fixed matrixes, and $K \in R^{q \times p}$ is variable matrix, so

$\underset{K}{g . r}[A+B K C]=\min \left\{\operatorname{rank}[A, B], \operatorname{rank}\left[\begin{array}{l}A \\ C\end{array}\right]\right\}$

, where, "g.r" denotes the generic rank of the matrix, i.e. it denotes the maximum rank of the matrix in the square brackets when the matrix $K$ changes.

\section{Description of problem}

The decentralized stabilization is to find $N$ local output control rules with dynamic compensator

$\left\{\begin{array}{l}E_{c_{i}} \dot{z}_{i}(t)=S_{i} z_{i}(t)+R_{i} y_{i}(t) \\ u_{i}(t)=Q_{i} z_{i}(t)+K_{i} y_{i}(t), i \in \underline{N}=\{1,2, \cdots, N\}\end{array}\right.$

, to make the closed-loop systems composed by the system (1) and the system (5) be regular, stable, and pulse-free. Where, $z_{i}(t) \in R^{n_{c_{i}}}$ is the state of the $i$ 'th controller, and the sizes of the matrixes $E_{c i}, S_{i}, R_{i}$, $Q_{i}$ and $K_{i}$ respectively are $m_{c_{i}} \times n_{c_{i}}, m_{c_{i}} \times n_{c_{i}}, m_{c_{i}} \times p_{i}, q_{i} \times n_{c_{i}}$, and $q_{i} \times p_{i}$.

The formula (5) can be simplified as

$\left\{\begin{array}{l}E_{c} \dot{z}(t)=S z(t)+R y(t) \\ u(t)=Q z(t)+K y(t)\end{array}\right.$

Where,

$$
\begin{aligned}
& E_{c} \square \text { block-diag }\left[E_{c_{1}}, E_{c_{2}}, \cdots, E_{c_{N}}\right] \\
& S \square \text { block-diag }\left[S_{1}, S_{2}, \cdots, S_{N}\right] \\
& Q \square \text { block-diag }\left[Q_{1}, Q_{2}, \cdots, Q_{N}\right] \\
& R \square \text { block-diag }\left[R_{1}, R_{2}, \cdots, R_{N}\right] \\
& K \square \text { block-diag }\left[K_{1}, K_{2}, \cdots, K_{N}\right] \\
& z \square\left[z_{1}{ }^{T}, z_{2}{ }^{T}, \cdots, z_{N}{ }^{T}\right]^{T}
\end{aligned}
$$

The whole closed-loop systems composed by the feedback rule (6) and the system (1) can be denoted as 
$\left[\begin{array}{cc}E & 0 \\ 0 & E_{c}\end{array}\right]\left[\begin{array}{c}\dot{x}(t) \\ \dot{z}(t)\end{array}\right]=\left[\begin{array}{cc}A+B K C & B Q \\ R C & S\end{array}\right]\left[\begin{array}{c}x(t) \\ z(t)\end{array}\right]$

, where,

$C^{T}=\left[\begin{array}{llll}C_{1}{ }^{T} & C_{2}{ }^{T} & \cdots & C_{N}{ }^{T}\end{array}\right], B=\left[\begin{array}{llll}B_{1}, & B_{2}, & \cdots, & B_{N}\end{array}\right]$

Note: $m_{c}=\sum_{i=1}^{N} m_{c_{i}}, n_{c}=\sum_{i=1}^{N} n_{c_{i}}$ and $r_{c}=\sum_{i=1}^{N} r_{c_{i}}$.

4. Main result

\subsection{Regularization and impulse-free}

The problems which should be first solved is the regularization and impulse-free of the system. According to the definition in Xie Xukai's article (Xie, 1986, P.185-188), the main idea is to compensate the system (1) to be a normal square system by the dynamic compensator, and then solve the problems of regularization and impulse-free of the square closed-loop systems.

The system (7) should fulfill the condition of regularization, i.e. this system should be square system, so the dimension of the designed compensator should fulfill

$n+n_{c}=m+m_{c}$

Theorem 1: For the system (1), the dynamic compensator (5) exists, and the sufficient and necessary condition to make the closed-loop systems (7) be regular and pulse-free is that, for any partition of the set $\underline{N}=\{1,2, \cdots, N\}$ into disjoint subsets $P=\left\{i_{1}, \cdots, i_{k}\right\}$ and $\bar{P}=\left\{i_{k+1}, \cdots, i_{N}\right\}$, the following inequality is true

$\operatorname{rank}\left[\begin{array}{ccc}0 & E & 0 \\ E & A & B_{P} \\ 0 & C_{\bar{P}} & 0\end{array}\right] \geq n+n_{c}+r-m_{P}-n_{\bar{P}}$

Define: $m_{P}=\sum_{i \in P} m_{c_{i}}, \quad n_{\bar{P}}=\sum_{i \in \bar{P}} n_{c_{i}}$.

Proof: By using the Lemma 2, the sufficient and necessary condition that the system (7) to be regular and pulse-free is the following equality holds:

$\operatorname{rank}\left[\begin{array}{cccc}0 & 0 & E & 0 \\ 0 & 0 & 0 & E_{c} \\ E & 0 & A+B K C & B Q \\ 0 & E_{c} & R C & S\end{array}\right]=n+n_{c}+r+r_{c}$

Supposed that

$$
\begin{aligned}
& \bar{E}_{c} \square \text { block-diag }\left[E_{c_{1}}, E_{c_{2}}, \cdots, E_{c_{N-1}}\right] \\
& \bar{S} \square \text { block-diag }\left[S_{1}, S_{2}, \cdots, S_{N-1}\right] \\
& \bar{Q} \square \text { block-diag }\left[Q_{1}, Q_{2}, \cdots, Q_{N-1}\right] \\
& \bar{R} \square \text { block-diag }\left[R_{1}, R_{2}, \cdots, R_{N-1}\right] \\
& \bar{K} \square \text { block-diag }\left[K_{1}, K_{2}, \cdots, K_{N-1}\right] \\
& \bar{C}^{T}=\left[\begin{array}{llll}
C_{1}{ }^{T} & C_{2}{ }^{T} & \cdots & C_{N-1}{ }^{T}
\end{array}\right], \bar{B}=\left[\begin{array}{lll}
B_{1}, & B_{2}, \cdots, & B_{N-1}
\end{array}\right]
\end{aligned}
$$

The left of the formula (10) can be written as 


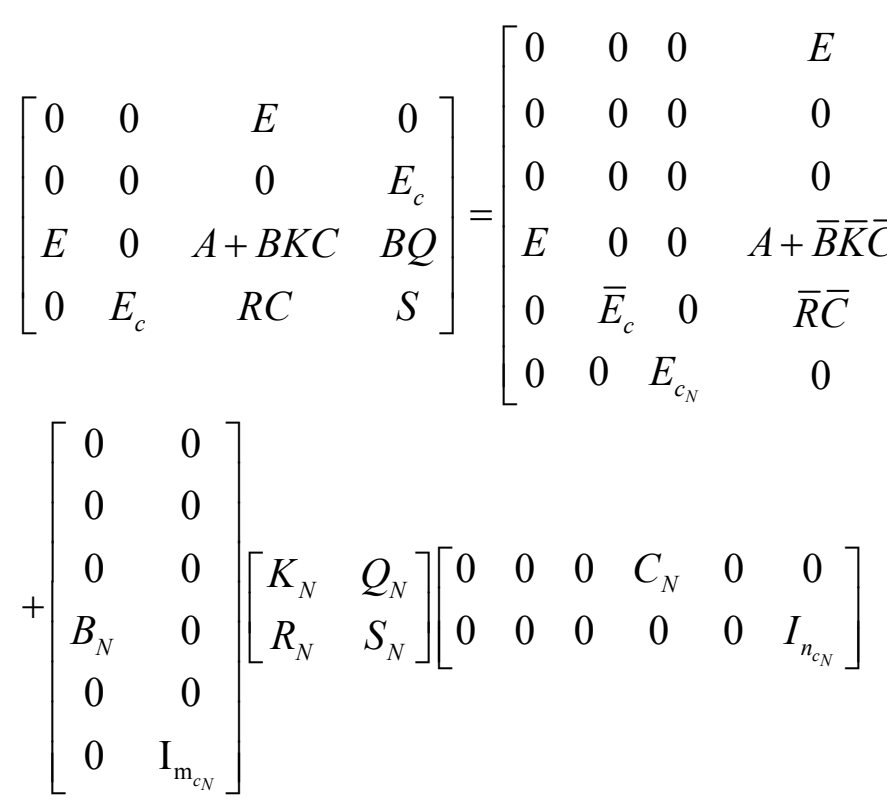

According to the Lemma 3, the sufficient and necessary condition that the formula (10) exists is

$\underset{\kappa}{g . r}\left[\begin{array}{cccc}0 & 0 & E & 0 \\ 0 & 0 & 0 & E_{c} \\ E & 0 & A+B K C & B Q \\ 0 & E_{c} & R C & S\end{array}\right]=\operatorname{rank}\left[\begin{array}{c}{\left[\begin{array}{ccccccc}0 & 0 & 0 & E & 0 & 0 & 0 \\ 0 & 0 & 0 & 0 & \bar{E}_{c} & 0 & 0 \\ 0 & 0 & 0 & 0 & 0 & E_{c_{N}} & 0 \\ E & 0 & 0 & A+\bar{B} \bar{K} \bar{C} & \bar{B} \bar{Q} & 0 & B_{N} \\ 0 & \bar{E}_{c} & 0 & \bar{R} \bar{C} & \bar{S} & 0 & 0\end{array}\right]+m_{c_{N}}} \\ \operatorname{rank}\left[\begin{array}{ccccc}0 & 0 & 0 & E & 0 \\ 0 & 0 & 0 & 0 & \bar{E}_{c} \\ E & 0 & 0 & A+\bar{B} \bar{K} \bar{C} & \bar{B} \bar{Q} \\ 0 & \bar{E}_{c} & 0 & \bar{R} \bar{C} & \bar{S} \\ 0 & 0 & E_{c_{N}} & 0 & 0 \\ 0 & 0 & 0 & C_{N} & 0\end{array}\right]+n_{c_{N}}\end{array}\right\}$

$=n+n_{c}+r+r_{c}$

The sufficient and necessary condition that the formula (12) exists is that

$\operatorname{rank}\left[\begin{array}{ccccccc}0 & 0 & 0 & E & 0 & 0 & 0 \\ 0 & 0 & 0 & 0 & \bar{E}_{c} & 0 & 0 \\ 0 & 0 & 0 & 0 & 0 & E_{c_{N}} & 0 \\ E & 0 & 0 & A+\bar{B} \bar{K} \bar{C} & \bar{B} \bar{Q} & 0 & B_{N} \\ 0 & \bar{E}_{c} & 0 & \bar{R} \bar{C} & \bar{S} & 0 & 0\end{array}\right]+m_{c_{N}} \geq n+n_{c}+r+r_{c}$

, and 
$\operatorname{rank}\left[\begin{array}{ccccc}0 & 0 & 0 & E & 0 \\ 0 & 0 & 0 & 0 & \bar{E}_{c} \\ E & 0 & 0 & A+\bar{B} \bar{K} \bar{C} & \bar{B} \bar{Q} \\ 0 & \bar{E}_{c} & 0 & \bar{R} \bar{C} & \bar{S} \\ 0 & 0 & E_{c_{N}} & 0 & 0 \\ 0 & 0 & 0 & C_{N} & 0\end{array}\right]+n_{c_{N}} \geq n+n_{c}+r+r_{c}$

, all exist.

By repeatedly using the decomposition of the formula (11) and the Lemma 3 to above two formulas, the sufficient and necessary condition that the formula (10) exist is

$\operatorname{rank}\left[\begin{array}{ccc}0 & E & 0 \\ E & A & B_{P} \\ 0 & C_{\bar{P}} & 0\end{array}\right] \geq n+n_{c}+r-m_{P}-n_{\bar{P}}, \forall P \subset \underline{N}$

, where, $m_{P}=\sum_{i \in P} m_{c_{i}}, n_{\bar{P}}=\sum_{i \in \bar{P}} n_{c_{i}}, P \bigcup \bar{P}=\underline{N}, P \cap \bar{P}=\Phi$, i.e. to any non-intersect divisions $P$ and $\bar{P}$, the above formula exists. End.

Note 1: In the condition (9), when $m=n$, there is $n_{c}=m_{P}+n_{\bar{P}}$, which is just the condition (2), i.e. the condition that the square descriptor decentralized control system is regular and impulse-free. That means the Theorem 1 keeps consistent with the sufficient and necessary condition that the square system is regular and impulse-free in form, i.e. it is the sufficient and necessary condition that the common descriptor system is regular and impulse-free.

\subsection{Stabilization}

Lemma 5 (G. C. Verghese, 1981, P.811-831): For the square system (1), the sufficient and necessary condition that $s \in C_{+e}$ is unstable finite decentralized fixed modes or pulse decentralized fixed modes is that, for any non-intersect divisions $P=\left\{i_{1}, \cdots, i_{k}\right\}$ and $\bar{P}=\left\{i_{k+1}, \cdots, i_{N}\right\}$ of the set $\underline{N}=\{1,2, \cdots, N\}$,

$\operatorname{rank}\left[\begin{array}{cc}s E-A & B_{P} \\ C_{\bar{P}} & 0\end{array}\right]<n$

, exists, and here, $C_{+e}=C_{+} \bigcup\{\infty\}$, and $C_{+}$denotes the right-half complex plane.

Lemma 6 (Z. W. Gao, 1997, P. 2520-2521): When and only when the system (1) has no unstable decentralized finite fixed modes and decentralized pulse fixed modes, the square system (1) could be real stable by the normal $\left(E_{c_{i}}=I\right)$ decentralized dynamic compensator, i.e. the corresponding closed-loop systems is interiorly real stable.

Lemma 7 (Yang, 2004): The sufficient and necessary condition that the square system (1) is closed and regular in the static output feedback is that, $s \in C$ (complex plane) exists, and for the certain one non-intersect divisions $P=\left\{i_{1}, \cdots, i_{k}\right\}$ and $\bar{P}=\left\{i_{k+1}, \cdots, i_{N}\right\}$ of the set $\underline{N}=\{1,2, \cdots, N\}$,

$\operatorname{rank}\left[\begin{array}{cc}s E-A & B_{P} \\ C_{\bar{P}} & 0\end{array}\right] \geq n$

Theorem 2: The sufficient and necessary condition that the descriptor decentralized system (1) is real stabilized by the descriptor dynamic compensator (5) is that, for any $s \in C_{+e}$, there is 
$\operatorname{rank}\left[\begin{array}{ccc}s E-A & B_{j} & B_{\varphi} \\ C_{\bar{\varphi}} & & \end{array}\right] \geq m$ and $\operatorname{rank}\left[\begin{array}{cc}s E-A & B_{\varphi} \\ C_{j} & \\ C_{\bar{\varphi}} & \end{array}\right] \geq n$

, where, $j \in \underline{N}=\{1,2, \cdots, N\}, \varphi$ and $\bar{\varphi}$ are any non-intersect divisions of the set $\underline{N}_{j}=\{1,2, \cdots, N\}-\{j\}$.

Prove: To study the stabilization of the rectangular system (1), the compensator should be used to compensate the system to be square system first, and here, the method to build the compensator can be described as follows.

One singular dynamic compensator is added to the $j$ 'th subsystem first, and for the convenience, supposed that the following singular compensator is added to the first subsystem.

$\left\{\begin{array}{l}E_{c_{1}} \dot{z}_{1}(t)=S_{1} z_{1}(t)+R_{1} y_{1}(t) \\ u_{1}(t)=Q_{1} z_{1}(t)+K_{1} y_{1}(t)\end{array}\right.$

To compensate the whole system into the square system, the corresponding closed-loop systems is

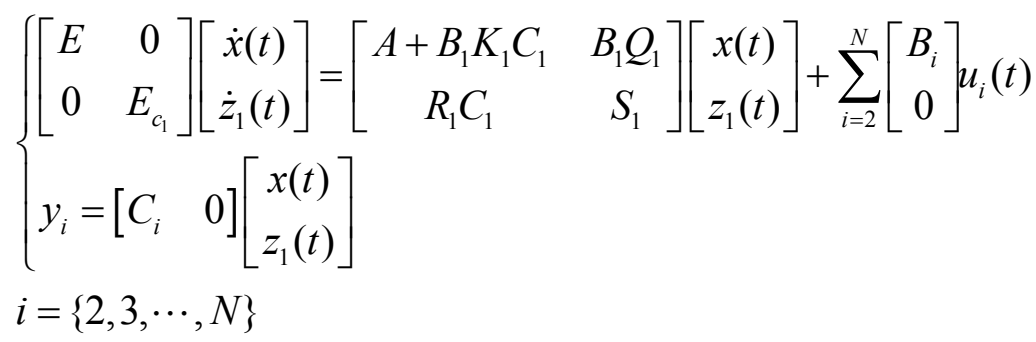

Supposed

$E^{\prime}=\left[\begin{array}{cc}E & 0 \\ 0 & E_{c_{1}}\end{array}\right], A^{\prime}=\left[\begin{array}{cc}A+B_{1} K_{1} C_{1} & B_{1} Q_{1} \\ R_{1} C_{1} & S_{1}\end{array}\right], B^{\prime}=\left[\begin{array}{c}B_{i} \\ 0\end{array}\right], C^{\prime}=\left[\begin{array}{ll}C_{i} & 0\end{array}\right]$

, and supposed that the dimension number of the sub-compensator fulfills

$n+n_{c_{1}}=m+m_{c_{1}}$

The stability of the closed-loop systems (21) is studied as follows.

From the Theorem 1, one dynamic compensator (20) exists to make the system (21) be closed and regular, i.e. all parameter matrixes in the system (21) is relatively fixed. By using the square system as references, the finite fixed modes of the system (21) could be defined as follows.

Definition 1: If $\max _{\operatorname{rank}}\left[s E^{\prime}-A^{\prime}+B^{\prime} K C^{\prime}\right]<n+n_{c_{1}}$ exists, and $s$ is finite, or $\operatorname{det}\left[s E^{\prime}-A^{\prime}+B^{\prime} K C^{\prime}\right]=0$

, so $S$ is one fixed modes or finite fixed modes of the system (21).

Definition 2: Supposed that $\left(s E^{\prime}-A^{\prime}\right)$ has infinite zero points, and if

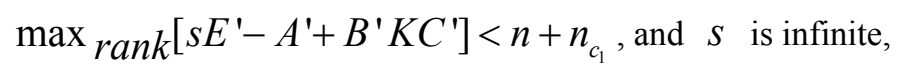

, the system (21) has the pulse fixed modes or the infinite fixed modes.

From the Lemma 5, for any $s \in C_{+e}$, when and only when

$\operatorname{rank}\left[\begin{array}{cc}s E-A & B_{P} \\ C_{\bar{P}} & 0\end{array}\right] \geq n$

, exists, the square system (1) has no unstable finite decentralized fixed modes and the impulse decentralized 
fixed modes. According to the Lemma 7, if the square system has no unstable finite decentralized fixed modes and the impulse decentralized fixed modes, the system certainly is closed-loop and regular in the feedback of static output.

According to above discussions, that the system (21) is closed-loop and regular could exist obviously, and based on that, the real stability is studied as follows (D. Wang, 1989, P.127-131), and $(N-1)$ normal dynamic compensators are added to the system (21).

$\left\{\begin{array}{l}\dot{z}_{i}(t)=S_{i} z_{i}(t)+R_{i} y_{i}(t) \\ u_{i}(t)=Q_{i} z_{i}(t)+K_{i} y_{i}(t), i \in \underline{N}_{1}=\{2,3, \cdots, N\}\end{array}\right.$

, here, $S_{i} \in R^{n_{c_{i}} \times n_{c_{i}}}, R_{i} \in R^{n_{c_{i}} \times p_{i}}, Q_{i} \in R^{q_{i} \times n_{c_{i}}}, K_{i} \in R^{q_{i} \times p_{i}}, i \in \underline{N}_{1}=\{2,3, \cdots, N\}$.

So the obtained closed-loop systems

$\left[\begin{array}{ccc}E & 0 & 0 \\ 0 & E_{c_{1}} & 0 \\ 0 & 0 & I\end{array}\right]\left[\begin{array}{c}\dot{x}(t) \\ \dot{z}_{1}(t) \\ \dot{\underline{z}}_{1}(t)\end{array}\right]=\left[\begin{array}{ccc}A+\sum_{i=1}^{N} B_{i} K_{i} C_{i} & B_{1} Q_{1} & \underline{B}_{1} \underline{Q}_{1} \\ R_{1} C_{1} & S_{1} & 0 \\ \underline{R}_{1} \underline{C}_{1} & 0 & \underline{S}_{1}\end{array}\right]\left[\begin{array}{c}x(t) \\ z_{1}(t) \\ \underline{z}_{1}(t)\end{array}\right]$

, is stable.

Where,

$$
\begin{aligned}
& \underline{S}_{1} \square \text { block-diag }\left[S_{2}, \cdots, S_{N}\right] \\
& \underline{Q}_{1} \square \text { block-diag }\left[Q_{2}, \cdots, Q_{N}\right] \\
& \underline{R}_{1} \square \text { block-diag }\left[R_{2}, \cdots, R_{N}\right] \\
& \underline{z}_{1} \square\left[z_{2}^{T}, \cdots, z_{N}{ }^{T}\right]^{T} \\
& \underline{C}_{1}^{T} \square\left[\begin{array}{lll}
C_{2}{ }^{T} & \cdots & C_{N}{ }^{T}
\end{array}\right], \underline{B}_{1} \square\left[\begin{array}{lll}
B_{2} & \cdots & B_{N}
\end{array}\right]
\end{aligned}
$$

Because the system (21) is closed and regular, so the closed-loop systems (25) must be regular, so the existence and uniqueness of the solution could be guaranteed. From the Lemma 5 and the Lemma 6, the sufficient and necessary condition that the square system (21) can be real stabilized by the normal compensator (24) is that the system (21) has no unstable decentralized finite fixed modes and the decentralized impulse fixed modes.

The sufficient and necessary condition that the system (21) has not unstable decentralized finite fixed modes and the decentralized impulse fixed modes is deduced as follows.

According to the definition of the fixed modes, for any $s \in C_{+e}$,

$$
\underset{k}{g . r}\left[s E^{\prime}-A^{\prime}+\sum_{i=2}^{N} B^{\prime} K_{i} C^{\prime}\right] \geq n+n_{c_{1}}
$$

, exists, and the system has no unstable decentralized finite fixed modes and the decentralized impulse fixed modes.

By repeatedly applying the Lemma 3,

$$
\operatorname{rank}\left[\begin{array}{cc}
s E^{\prime}-A^{\prime} & B_{\varphi_{1}} \\
C_{\bar{\varphi}_{1}} & 0
\end{array}\right] \geq n+n_{c_{1}}
$$

Here, $\varphi$ and $\bar{\varphi}$ are certain non-intersect divisions $\varphi_{1}=\left\{i_{2}, \cdots, i_{k}\right\}$ and $\bar{\varphi}_{1}=\left\{i_{k+1}, \cdots, i_{N}\right\}$.

That is just equivalent with the Lemma 5 .

The formula (27) can be denoted as follows. 


$$
\begin{aligned}
& \operatorname{rank}\left[\begin{array}{cc}
s E^{\prime}-A^{\prime} & B_{\varphi_{1}} \\
C_{\bar{\varphi}_{1}} & 0
\end{array}\right]=\operatorname{rank}\left[\begin{array}{ccc}
s E-A-B_{1} K_{1} C_{1} & -B_{1} Q_{1} & B_{\varphi_{1}} \\
-R_{1} C_{1} & s E_{c_{1}}-S_{1} & 0 \\
C_{\bar{\varphi}_{1}} & 0 & 0
\end{array}\right] \\
& =\operatorname{rank}\left\{\left[\begin{array}{ccc}
s E-A & 0 & B_{\varphi_{1}} \\
0 & s E_{c_{1}} & 0 \\
C_{\bar{\varphi}_{1}} & 0 & 0
\end{array}\right]+\left[\begin{array}{cc}
B_{1} & 0 \\
0 & \mathrm{I}_{\mathrm{m}_{c_{1}}} \\
0 & 0
\end{array}\right]\left[\begin{array}{cc}
-K_{1} & -Q_{1} \\
-R_{1} & -S_{1}
\end{array}\right]\left[\begin{array}{ccc}
C_{1} & 0 & 0 \\
0 & \mathrm{I}_{n_{c_{1}}} & 0
\end{array}\right]\right\}
\end{aligned}
$$

From the Lemma 3 and the formula (28),

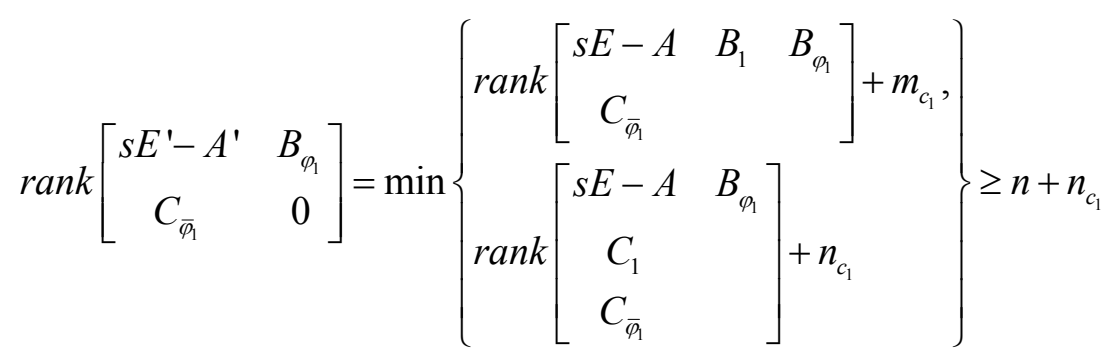

So the sufficient and necessary condition that the formula (29) exists is that

$\operatorname{rank}\left[\begin{array}{ccc}s E-A & B_{1} & B_{\varphi_{1}} \\ C_{\bar{\varphi}_{1}} & & \end{array}\right] \geq m$

, and

$\operatorname{rank}\left[\begin{array}{cc}s E-A & B_{\varphi_{1}} \\ C_{1} & \\ C_{\bar{\varphi}_{1}} & \end{array}\right] \geq n$

, all exist.

So the sufficient and necessary condition that the system (21) has no unstable decentralized finite fixed modes and the decentralized impulse fixed modes can be obtained, i.e. the sufficient and necessary condition that the system (1) can be real stabilized by the compensator (20) and the compensator (24).

And the compensator (20) and the compensator (24) can be written as following form.

$$
\left\{\begin{array}{l}
{\left[\begin{array}{cc}
E_{c_{1}} & 0 \\
0 & I
\end{array}\right]\left[\begin{array}{l}
\dot{z}_{1}(t) \\
\dot{z}_{1}(t)
\end{array}\right]=\left[\begin{array}{cc}
S_{1} & 0 \\
0 & \underline{S}_{1}
\end{array}\right]\left[\begin{array}{c}
z_{1}(t) \\
z_{1}(t)
\end{array}\right]+\left[\begin{array}{cc}
R_{1} & 0 \\
0 & \underline{R}_{1}
\end{array}\right]\left[\begin{array}{l}
y_{1}(t) \\
y_{1}(t)
\end{array}\right]} \\
{\left[\begin{array}{l}
u_{1}(t) \\
\underline{u}_{1}(t)
\end{array}\right]=\left[\begin{array}{cc}
Q_{1} & 0 \\
0 & \underline{Q}_{1}
\end{array}\right]\left[\begin{array}{l}
z_{1}(t) \\
\underline{z}_{1}(t)
\end{array}\right]+\left[\begin{array}{cc}
K_{1} & 0 \\
0 & \underline{K}_{1}
\end{array}\right]\left[\begin{array}{l}
y_{1}(t) \\
\underline{y}_{1}(t)
\end{array}\right]}
\end{array}\right.
$$

, where, the definitions of $\underline{\dot{z}}_{1}, \underline{S}_{1}, \underline{R}_{1}, \underline{Q}_{1}$ are same in the former of the article, and in addition

$$
\underline{K}_{1} \square \text { block-diag }\left[K_{2}, \cdots, K_{N}\right]
$$

$\underline{y}_{1} \square\left[y_{2}{ }^{T}, \cdots, y_{N}{ }^{T}\right]^{T}$

$\underline{u}_{1} \square\left[u_{2}^{T}, \cdots, u_{N}^{T}\right]^{T}$

In this way, the obtained dynamic compensator (32) can real stabilize the rectangular singular system (1), and obviously, the compensator (32) keeps consistent with the compensator (5) in form.

By the same way, the sufficient and necessary condition that one singular dynamic compensator is added to the 
$j$ 'th subsystem of the system could be deduced.

End.

Note 2: In the Theorem 2, when $m=n$, the formula (19) is equivalent to the Lemma 5 in meaning.

\section{Example}

For one double-channel system,

$$
\left\{\begin{array}{l}
E \dot{x}(t)=A x(t)+\sum_{i=1}^{2} B_{i} u_{i}(t) \\
y_{i}(t)=C_{i} x(t), i \in \underline{N}=\{1,2\}
\end{array}\right.
$$

, where,

$$
E=\left[\begin{array}{lll}
0 & 0 & 1 \\
1 & 0 & 0 \\
0 & 1 & 0 \\
0 & 0 & 0
\end{array}\right], A=\left[\begin{array}{lll}
1 & 0 & 0 \\
1 & 0 & 0 \\
0 & 1 & 1 \\
0 & 0 & 1
\end{array}\right], B_{1}=\left[\begin{array}{l}
0 \\
1 \\
0 \\
0
\end{array}\right]
$$

\section{Conclusions}

The dynamic compensator is adopted to change the rectangular descriptor decentralized system into normal square system, and the corresponding closed-loop systems is studied in this article, and the sufficient and necessary condition that the closed-loop systems is regular and impulse-free is obtained, which keeps consistent with the corresponding sufficient and necessary condition of the square system, as seen in the formula (9). For the stability, the singular dynamic compensator is added to one subsystem, and then the normal dynamic compensator is added to corresponding closed-loop systems, in order to stabilize the rectangular singular system, and the sufficient and necessary condition of the corresponding square system keeps consistent in form, as seen in the formula (19). In this way, the whole system is not only gradually stable, but also has not destructive pulse behaviors, which is very important to perfect the descriptor decentralized system theory and apply the descriptor decentralized system in the practice.

\section{References}

D. Wang and C. B. Soh. (1989). On regularizing singular systems by decentralized output feedback. IEEE Trans. Automat. Contr. Vol. 44. P.127-131.

G. C. Verghese, B.C. Levy, and T. Kailath. (1981). A descriptor state-space for singular systems. IEEE Trans. Automat. Contr. No. 26 (4). P.811-831.

Hou M. (2004). Controllability and elimination of impulsive modes in descriptor systems. IEEE Trans. Automatica, Contr. No. 49(10). P.1723-1727.

Ishihara J Y, Terra M H. (2001). Impulse controllability and observability of rectangular descriptor systems. IEEE Trans. Automat. Contr. No. 46(6). P.991-994.

L. Dai. (1989). Singular Control Systems-Lecture Notes in Control and Information Science. Berlin, Germany: Springer-Verlag.

Q. L. Zhang. (1989). Algebraical Characterizations of Fixed Modes in Linear Decentralized Descriptor Systems. Conference on Decision and Control. P.866-871.

S. H. Wang and E. J. Davison. (1973). On the stabilization of decentralized control systems. IEEE Trans. Automat. Contr. Vol. AC-18. P. 473-478. Oct. 1973.

T. N. Chang and E. J. Davison. (2001). Decentralized control of descriptor systems. IEEE Trans. Automat. Contr. Vol. AC-46. P. 1589-1595. Oct. 2001.

T. N. Chang and E. J. Davison. (1986). Decentralized control of descriptor type systems. Proc. of the 25th IEEE Conference on Decision and Control. P.1276-1181.

Xie, Xukai et al. (1995). Uniform Judgement of the Fixed Mode of the Descriptor Decentralized Control System. Acta Automatic Sinica. No. 21(2). P.145-153.

Xie, Xukai \& Jin, Haiying. (1986). Fixed Mode of the Decentralized Control System. Acta Automatic Sinica. No. 12(2). P.185-188. 
X. K. Xie. (1988). On fixed modes in singular systems. Proc. of American Control Conference. P. 1550-1551. Yang, Dongmei \& Zhang, Qingling et al. (2004). Descriptor System. Beijing: Science Press.

Zhang, Guoshan. (2006). Regularization and Pole-placement of Descriptor Systems by Dynamic Compensation. Control and Decision. No.1.

Z. W. Gao and X. L. Wang. (1997). Internal properness and stability in singular decentralized control systems. Proc. of American Control Conference. P. 2520-2521. 